\title{
A simple classification system (the Tree flowchart) for breast MRI can reduce the number of unnecessary biopsies in MRI-only lesions
}

\author{
Ramona Woitek $^{1}$ - Claudio Spick ${ }^{1} \cdot$ Melanie Schernthaner ${ }^{1} \cdot$ Margaretha Rudas $^{2}$ • \\ Panagiotis Kapetas $^{1}$ • Maria Bernathova ${ }^{1}$ • Julia Furtner ${ }^{1}$ - Katja Pinker ${ }^{1}$. \\ Thomas H. Helbich ${ }^{1}$ • Pascal A. T. Baltzer ${ }^{1}$
}

Received: 2 November 2016 / Revised: 9 January 2017 / Accepted: 19 January 2017 / Published online: 8 March 2017

(C) The Author(s) 2017. This article is published with open access at Springerlink.com

\begin{abstract}
Objectives To assess whether using the Tree flowchart obviates unnecessary magnetic resonance imaging (MRI)-guided biopsies in breast lesions only visible on MRI.

Methods This retrospective IRB-approved study evaluated consecutive suspicious (BI-RADS 4) breast lesions only visible on MRI that were referred to our institution for MRIguided biopsy. All lesions were evaluated according to the Tree flowchart for breast MRI by experienced readers. The Tree flowchart is a decision rule that assigns levels of suspicion to specific combinations of diagnostic criteria. Receiver operating characteristic (ROC) curve analysis was used to evaluate diagnostic accuracy. To assess reproducibility by kappa statistics, a second reader rated a subset of 82 patients. Results There were 454 patients with 469 histopathologically verified lesions included (98 malignant, 371 benign lesions). The area under the curve (AUC) of the Tree flowchart was 0.873 (95\% CI: 0.839-0.901). The inter-reader agreement was almost perfect (kappa: 0.944; 95\% CI 0.889-0.998). ROC analysis revealed exclusively benign lesions if the Tree node was $\leq 2$, potentially avoiding unnecessary biopsies in 103 cases $(27.8 \%)$.
\end{abstract}

Electronic supplementary material The online version of this article (doi:10.1007/s00330-017-4755-6) contains supplementary material, which is available to authorized users.

Pascal A. T. Baltzer

pascal.baltzer@meduniwien.ac.at

Department of Biomedical Imaging and Image-Guided Therapy, Medical University of Vienna, Vienna, Austria

2 Clinical Institute of Pathology, Medical University of Vienna, Vienna, Austria
Conclusions Using the Tree flowchart in breast lesions only visible on MRI, more than $25 \%$ of biopsies could be avoided without missing any breast cancer.

Key Points

- The Tree flowchart may obviate $>25 \%$ of unnecessary MRIguided breast biopsies.

- This decrease in MRI-guided biopsies does not cause any false-negative cases.

- The Tree flowchart predicts $30.6 \%$ of malignancies with $>98 \%$ specificity.

- The Tree's high specificity aids in decision-making after benign biopsy results.

Keywords Magnetic resonance imaging - Breast cancer Scoring system $\cdot$ Image-guided biopsy $\cdot$ ROC curve

$\begin{array}{ll}\begin{array}{l}\text { Abbreviations } \\ \text {-LR } \\ + \text { LR }\end{array} & \begin{array}{l}\text { Negative likelihood ratio } \\ \text { Positive likelihood ratio } \\ \text { ACR BI-RADS }\end{array} \\ & \begin{array}{l}\text { American College of Radiology } \\ \text { Breast Imaging and Reporting } \\ \text { Data System }\end{array} \\ \text { ADC } & \text { Apparent diffusion coefficient } \\ \text { AUC } & \text { Area under the curve } \\ \text { ben } & \text { Benign } \\ \text { CI } & \text { Confidence interval } \\ \text { DCIS } & \text { Ductal carcinoma in situ } \\ \text { DWI } & \text { Diffusion-weighted imaging } \\ \text { EUSOMA } & \text { European Society of } \\ \text { FA } & \text { Breast Cancer Specialists } \\ \text { FFE } & \text { Flip angle } \\ \text { FLASH } & \text { Fast field echo } \\ & \text { Fast low angle shot }\end{array}$




$\begin{array}{ll}\text { FOV } & \text { Field of view } \\ \text { FS } & \text { Fat saturation } \\ \text { high-risk } & \text { High-risk lesion } \\ \text { mal } & \text { Malignant } \\ \text { mm } & \text { Millimetre } \\ \text { MRI } & \text { Magnetic resonance imaging } \\ \text { ms } & \text { Millisecond } \\ \mathrm{n} & \text { Number } \\ \text { NPV } & \text { Negative predictive value } \\ \text { ROC } & \text { Receiver operating characteristic } \\ \sum & \text { SumSD, Standard deviation } \\ \text { sens } & \text { Sensitivity } \\ \text { SPAIR } & \text { Spectral attenuated } \\ & \text { inversion recovery } \\ \text { spec } & \text { Specificity } \\ \text { ST } & \text { Slice thickness } \\ \text { STIR } & \text { Short tau inversion recovery } \\ \text { T } & \text { Tesla } \\ \text { TE } & \text { Echo time } \\ \text { TI } & \text { Inversion time } \\ \text { TIRM } & \text { Turbo inversion recovery } \\ & \text { magnitude } \\ \text { TR } & \text { Repetition time } \\ \text { VABB } & \text { Vacuum-assisted } \\ & \text { breast biopsy } \\ \end{array}$

\section{Introduction}

Breast lesions rated as suspicious for cancer according to the American College of Radiology Breast Imaging and Reporting Data System (ACR BI-RADS) (e.g. assigned an ACR BI-RADS $\geq 4$ category) that were detected on breast magnetic resonance imaging (MRI) require tissue sampling and histopathological workup [1-3]. Unless these lesions are visible on other imaging modalities, they require dedicated MRI-guided, vacuum-assisted breast biopsy (VABB) to provide representative tissue sampling [1, 2, 4]. As reflected by positive predictive values of MRI-guided biopsies below $50 \%$ in the literature [5-8], a relevant number of benign lesions visible on MRI undergo unnecessary VABBs that can potentially be avoided. MR-guided VABB is a safe and accurate procedure in the diagnostic workup, but its application is limited by availability, relatively high costs compared to other biopsy techniques, and the necessity to administer gadolinium-containing contrast agent intravenously [9]. Although minimally invasive, MR-guided biopsies carry a low risk for complications, such as infection or bleeding [9]. There is general consent that unnecessary biopsies should be avoided [10] by ruling out malignancy based on imaging features. Numerous efforts have been made to decrease the number of false-positive results in standard breast MRI using additional imaging techniques, such as diffusion-weighted imaging (DWI), MR spectroscopy (MRS) and positron emission tomography (PET), or sophisticated evaluation of dynamic contrast-enhanced MRI [11-17]. Although these approaches may increase specificity, there are several issues regarding standardization, as well as time and cost effectiveness. It would be thus desirable if such an increase in specificity could be achieved using standard breast MRI sequences only.

To report imaging features and indicate suspicion for malignancy, ACR BI-RADS is the most widely used standard. It facilitates communication among physicians with its structured common language and standardized terminology for image interpretation and reporting. However, it lacks precise rules according to which to assign imaging features (i.e. lesion morphology and functional contrast enhancement kinetics) to diagnostic categories. Therefore, using ACR BI-RADS, the inter-reader agreement remains moderate, diagnostic accuracy is variable, a relevant number of unnecessary biopsies are performed in benign lesions, the inter-reader agreement remains moderate, and diagnostic accuracy is variable [18-22].

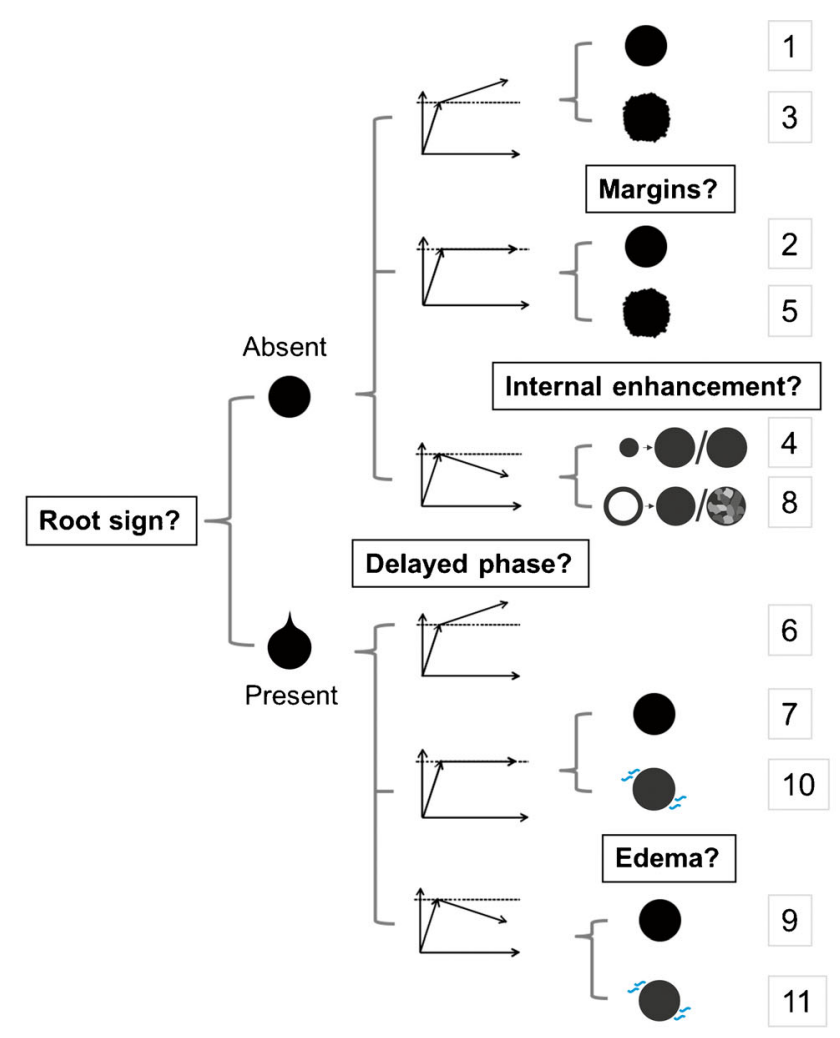

Legend: : perifocal edema present, $O$ : edema absent
: cetrifugal or homogeneous enhancement

Fig. 1 Tree flowchart following the description by Marino et al. [24]. Terminal nodes are hierarchically ordered (1-11) and represent increasing probabilities of malignancy 
To complement the ACR BI-RADS lexicon and to increase specificity, Baltzer et al. proposed a simple classification system (the Tree flowchart) for breast MRI to differentiate benign and malignant lesions on breast MRI [23]. The Tree flowchart combines five diagnostic criteria (the root sign, enhancement kinetics, lesion margins, internal enhancement pattern and ipsilateral oedema; Fig. 1, Table 1) to assign a diagnostic score to each lesion, indicating the likelihood of malignancy. These five criteria were selected from a larger pool of 17 criteria, based on their representation of possibly malignant lesion features [25-28] and their non-redundancy [23]. Neither the initial exploratory evaluation of the Tree flowchart [23] nor the subsequent independent validation study [24] specifically addressed a defined clinical setting where the Tree could improve clinical management.

Thus, the aim of this study was to assess whether using the Tree flowchart obviates unnecessary MRI-guided biopsies in MRI-only breast lesions.

Table 1 The morphological and kinetic criteria included in the Tree flowchart

\begin{tabular}{|c|c|c|}
\hline Root sign & $\begin{array}{l}\text { The 'root sign' is characterized by a root-like } \\
\text { tip at the lesion margin, that is surrounded } \\
\text { on both sides by concave margin segments } \\
\text { (red). The 'root' sign can vary from a single } \\
\text { tip to multiple spiculations. The root sign is } \\
\text { suspicious for malignancy }\end{array}$ & - \\
\hline $\begin{array}{l}\text { Persistent } \\
\text { enhancement }\end{array}$ & $\begin{array}{l}\text { Progressive rise in the enhancement curve } \\
\text { on early and delayed phase T1-weighted } \\
\text { images is considered not suspicious. }\end{array}$ & \\
\hline $\begin{array}{l}\text { Plateau } \\
\text { enhancement }\end{array}$ & $\begin{array}{l}\text { No further rise of signal intensity occurs on } \\
\text { delayed phase } \mathrm{T} 1 \text {-weighted images after } \\
\text { early signal increase is considered } \\
\text { equivocal. }\end{array}$ & \\
\hline Washout & $\begin{array}{l}\text { Decline in signal intensity on delayed } \\
\text { images after early signal increase is } \\
\text { considered suspicious. }\end{array}$ & \\
\hline Margins & $\begin{array}{l}\text { General characteristic of the margin of a } \\
\text { lesion: smooth (not suspicious) or irregular } \\
\text { (suspicious). Note that in a generally } \\
\text { smoothly bordered lesion the root sign can } \\
\text { be present. }\end{array}$ & \\
\hline $\begin{array}{l}\text { Internal } \\
\text { enhancement } \\
\text { pattern }\end{array}$ & $\begin{array}{l}\text { The general pattern of enhancement of a } \\
\text { lesion is dichotomized into suspicious } \\
\text { (centripetal/rim enhancement or } \\
\text { inhomogeneous) or not suspicious } \\
\text { (centrifugal or homogeneous } \\
\text { enhancement). }\end{array}$ & \\
\hline $\begin{array}{l}\text { Ipsilateral } \\
\text { edema }\end{array}$ & $\begin{array}{l}\text { High water-like signal on T2-weighted } \\
\text { images not caused by ductectasia } \\
\text { surrounding a lesion or diffusely distributed } \\
\text { in the breast harbouring a lesion is } \\
\text { suggestive of breast cancer. Entirely absent } \\
\text { or bilateral diffuse edema is rated negative. }\end{array}$ & \\
\hline
\end{tabular}


Fig. 2 Example of a non-mass lesion: ductal carcinoma in situ (DCIS) Grade 3, presenting as a non-mass lesion without the root sign, with plateau enhancement during the delayed phase and with irregular margins. Based on the Tree flowchart (Fig. 1), the described characteristics resulted in a node 5 rating where malignancy cannot be ruled out
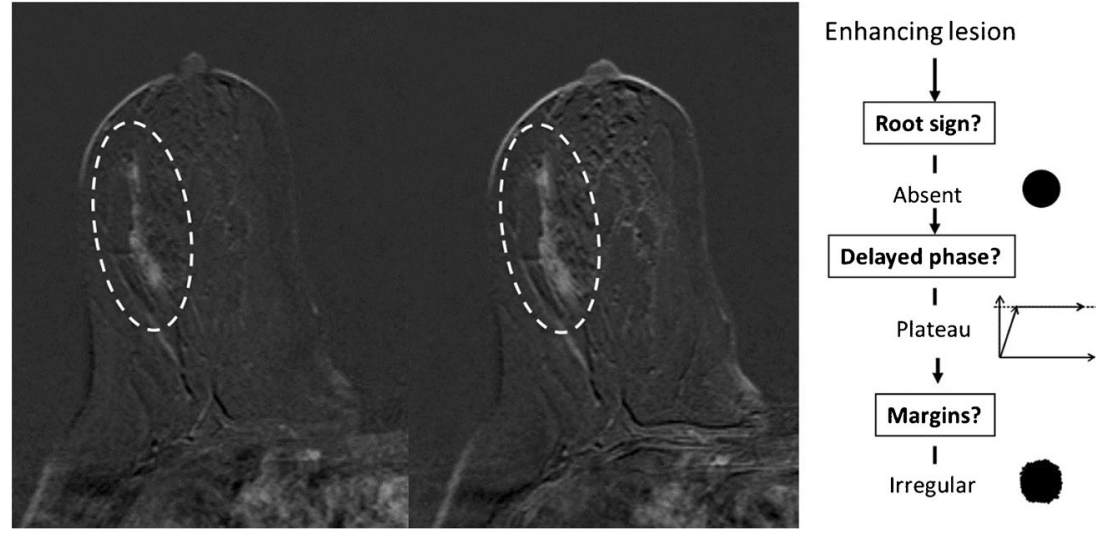

\section{Materials and methods}

\section{Study design}

This cross-sectional, retrospective, single-centre study was approved by our institutional review board (IRB). The necessity for informed consent was waived. There were 454 consecutive patients (mean age $52 \pm 13$ years) with 469 breast lesions visible only on MRI undergoing MRI-guided VABB and/or surgical biopsy at our institution from January 2006 to December 2013 who were included. There was a partial overlap of the data analysed in this study with two prior publications $[11,22]$. However, study rationale and results differed between the studies.

\section{Imaging and MRI-guided biopsies}

Based on our ethical review board-approved study protocol, a database was populated with the results obtained during retrospective readings of diagnostic breast MRI scans. MRI scans were performed in accordance with the EUSOMA (European Society of Breast Cancer Specialists) recommendations [2] at different referring institutions, on 1.5- and 3Tesla ( $\mathrm{T}$ ) units of different vendors, using dedicated breast coils: seven patients $(0.2 \%)$ were scanned at 1.0-T, 422 patients $(93 \%)$ at $1.5-\mathrm{T}$ and 25 patients $(5.5 \%)$ at $3.0-\mathrm{T}$. There were 383 examinations (84.4\%) acquired using Siemens MAGNETOM scanners (Erlangen, Germany): Avanto (261 patients, 57.5\%), Symphony (50 patients, $11 \%$ ), Essenza (21 patients, 4.6\%), Espree (18 patients, 4\%), Trio (17 patients, 37.4\%), Harmony (seven patients, $1.5 \%$ ), Verio (three patients, $0.7 \%$ ), Aera (three patients, $0.7 \%$ ), Skyra (one patient, $0.2 \%$ ) and Vision (one patient, $0.2 \%)$. Seventy-one examinations $(15.6 \%)$ were acquired on Philips Scanners (Philips Medical Systems, Best, The Netherlands): Intera (61 patients, 13.4\%), Achieva (seven patients, $1.5 \%$ ) and Ingenia (four patients, $0.9 \%$ ). The following contrast agents were applied intravenously at dosages of $0.1 \mathrm{mmol}$ gadolinium $/ \mathrm{kg}$ body weight: Dotarem (gadoterate meglumine/Guerbet, Villepinte,
Fig. 3 Apocrine ductal carcinoma in situ (DCIS) Grade 2 presenting as a mass lesion with the root sign, delayed plateau enhancement and perifocal oedema, resulting in a classification as Tree node 10 . Representative axial slices of the T1-weighted, non-enhanced sequence (a), a T2 TIRM sequence (b), early (c) and delayed (d) post-contrast T1weighted sequences are shown
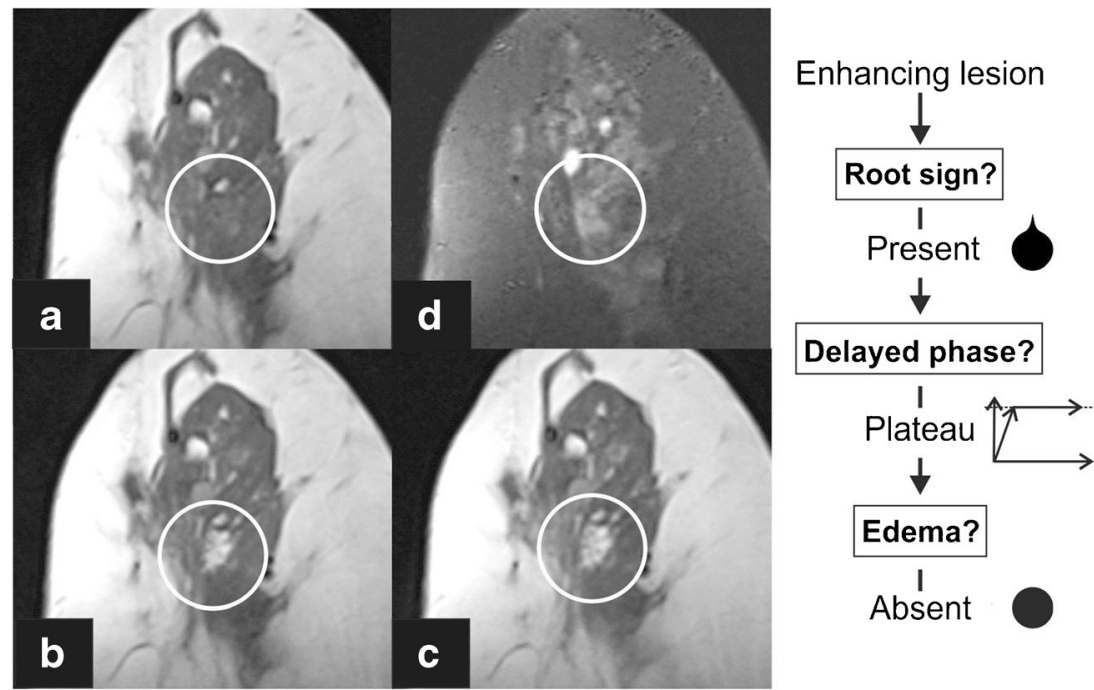
Fig. 4 Benign columnar cell change and flat epithelial hyperplasia were diagnosed in this mass lesion showing plateau enhancement and smooth margins without the root sign, thus resulting in a Tree node 2. Biopsy could have been avoided with the Tree classification in this lesion. Representative slices of the T2weighted (a) and the unenhanced T1-weighted sequences (b) are shown, as well as early (c) and delayed (d) subtractions of contrast-enhanced T1-weighted sequences
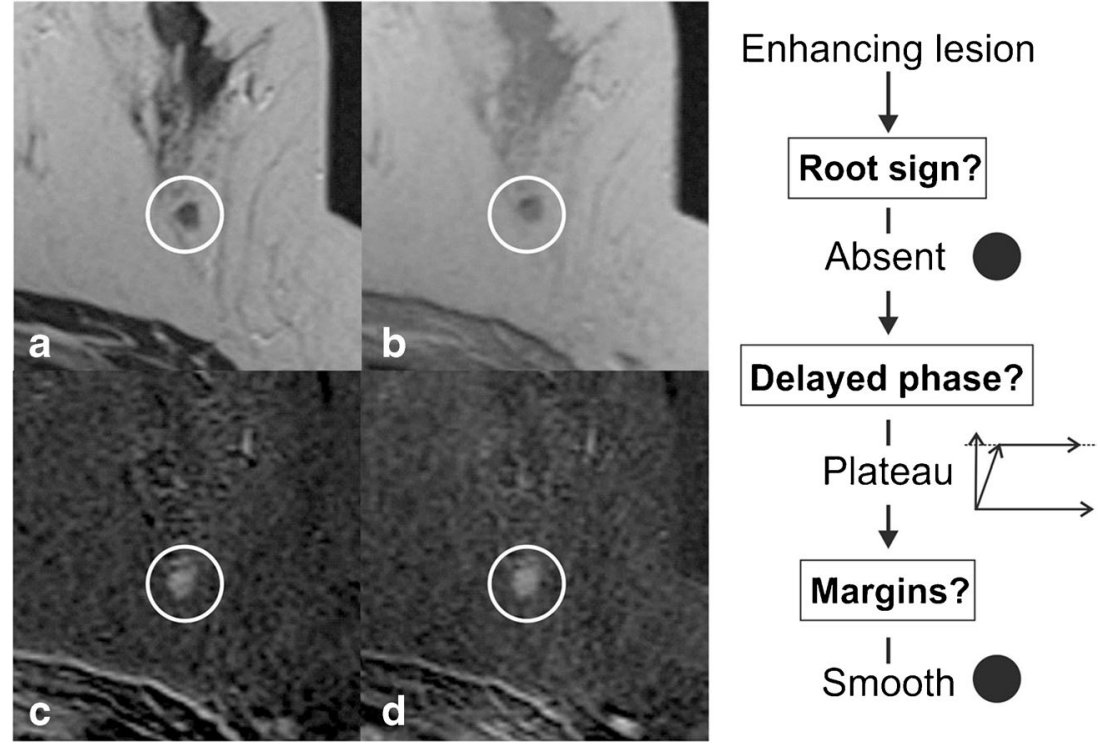

France), Multihance (gadobenate dimeglumine/Bracco Diagnostics, Princeton, NJ, USA), Gadovist (gadobutrol/ Bayer Pharmaceuticals, Berlin, Germany), Prohance (gadoteridol/Bracco Diagnostics, Princeton, NJ, USA) and Omniscan (gadodiamide/ GE Healthcare, Princeton, NJ, USA). The detailed sequence parameters of the T2weighted and the dynamic T1-weighted sequences are shown in ESM 1.

All lesions classified as BI-RADS 4 (suspicious) were biopsied under MRI-guidance at our institution on a 1.5-T system (Avanto, Siemens) using a dedicated double breast imaging and intervention coil (InVivo, Philips).

Written informed consent for the MRI-guided VABB was obtained from all patients in advance.

MRI-guided VABBs were performed as previously described [22]. For lesion localization, a shortened imaging protocol was acquired: before and after the application of intravenous (IV) contrast agent, dynamic, contrast-enhanced, T1-weighted gradient echo sequences were acquired and subtraction images were obtained. The T1weighted sequence was repeated to verify needle positioning during the biopsy. After VABB, the biopsy sites were marked with MRI-compatible radiopaque clip markers. An experienced board-certified breast pathologist (M.R.) performed histopathological tissue analyses and applied the B classification for diagnosis [29]. Histopathological results and the MRI were compared in interdisciplinary consensus. In cases of discrepancy between imaging results and histopathology, and in lesions with uncertain malignant potential (B3), surgical biopsy was performed, after wire localization, by board-certified, experienced breast surgeons. In case of a benign finding at histopathology, the patients were followed up with breast MRI for at least 12 months.

\section{Data analysis}

All examinations were analysed by an experienced breast imaging radiologist (P.A.B, $>10$ years of breast MRI experience), and 82 consecutive cases were read by a second reader independently (C.S., radiology resident) to assess inter-reader
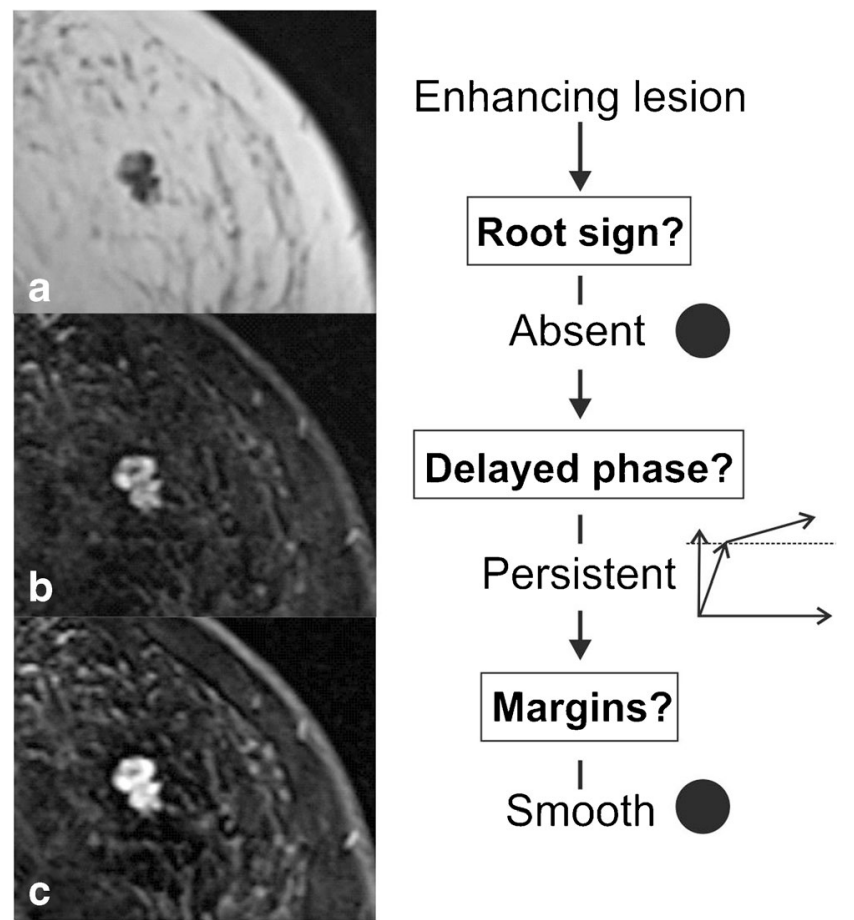

Fig. 5 Fibroadenomatous hyperplasia presenting as a mass lesion with persistent enhancement and smooth borders without the root sign (note the lobulations with konvex tips that do not fulfill the criteria of the root sign). The lesion was thus classified as node 1 and could have been identified as benign using the Tree flowchart. Representative slices of a non-enhanced T1-weighted sequence (a) and subtractions of the early (b) and delayed (c) post-contrast T1-weighted sequences are shown 
Table 2 Size distributions in mass and non-mass lesions stratified by histopathological results

\begin{tabular}{|c|c|c|c|c|c|}
\hline Lesion type & $\mathrm{n}$ & Mean diameter (mm) & Histology & $\mathrm{n}$ & Mean diameter $(\mathrm{mm})$ \\
\hline \multirow[t]{2}{*}{ Mass lesions } & \multirow[t]{2}{*}{270} & \multirow[t]{2}{*}{$9 \pm 5(\mathrm{SD})$} & Malignant & 68 & $11 \pm 6(\mathrm{SD})$ \\
\hline & & & Benign & 202 & $9 \pm 5(\mathrm{SD})$ \\
\hline \multirow[t]{2}{*}{ Non-mass lesions } & \multirow[t]{2}{*}{199} & \multirow[t]{2}{*}{$26 \pm 15$ (SD) } & Malignant & 30 & $35 \pm 16$ (SD) \\
\hline & & & Benign & 169 & $24 \pm 14$ (SD) \\
\hline \multirow[t]{2}{*}{ Total } & \multirow[t]{2}{*}{469} & \multirow[t]{2}{*}{$16 \pm 13(\mathrm{SD})$} & Malignant & 98 & $18 \pm 15$ (SD) \\
\hline & & & Benign & 371 & $16 \pm 12(\mathrm{SD})$ \\
\hline
\end{tabular}

$S D$ standard deviation, $n$ number

agreement. Both readers were blinded to the final histopathological diagnosis and previous reading results, if available.

The readers were asked to classify all identified lesions following the Tree flowchart. This simple classification system is based on five morphological and kinetic criteria (root sign, contrast enhancement kinetics, lesion margins, internal enhancement patterns and oedema; Table 1 and Fig. 1) evaluated on T2-weighted sequences and dynamic, contrast-enhanced, T1-weighted sequences. The Tree flowchart contains 11 assignment categories that correspond to an increasing probability of malignancy ( 1 = lowest, cancer very unlikely, to 11 = highest, cancer very likely; Fig. 1) [23, 24]. Examples of lesions are given in Figs. 2, 3, 4 and 5. A diagnostic category was chosen for each lesion by following the Tree flowchart and was noted in a spreadsheet (Fig. 1).

\section{Statistical analyses}

SPSS 23.0 (SPSS, IBM, Chicago, IL, USA) and MedCalc 15 (MedCalc software bvba, Ostend, Belgium) were used for statistical analyses. Inter-reader agreement in the assigned Tree categories was assessed using kappa statistics. A receiver operating characteristic (ROC) analysis was performed and the area under the ROC curve was measured to determine overall diagnostic performance. Sensitivity, specificity and likelihood ratios were calculated at different cut-off values. P-values $\leq 0.05$ were considered statistically significant. Two cut-off values, to rule out and rule in malignancy, were selected according to the calculated sensitivities, specificities and numbers of falsepositive and false-negative results (low cut-off value with highest sensitivity and lowest false-positive results; high cutoff value with high specificity and low false-negative results).

\section{Results}

\section{Patients and lesions}

Ninety-eight of the included 469 breast lesions (20.9\%) were histopathologically diagnosed as malignant, and $371(79.1 \%)$ lesions as benign.
There were 270 lesions $(57.6 \%)$ that presented as masses, whereas 199 (42.4\%) were non-mass lesions. Of all 270 mass lesions, 68 (25.2\%) were malignant and 202 (74.8\%) benign. Of the 199 non-mass lesions, 30 (15.1\%) were malignant and $169(84.9 \%)$ benign. Detailed size descriptions and descriptive statistics of histopathological diagnoses are given in Tables 2 and 3 .

\section{ROC curve analyses}

Using the Tree flowchart for all lesions, the overall accuracy represented by the area under the ROC curve (AUC)

Table 3 Descriptive statistics for histopathological diagnoses among all lesions stratified by Tree nodes

\begin{tabular}{|c|c|c|c|c|c|c|}
\hline Node & & Benign & High-risk & DCIS & Invasive cancer & Total \\
\hline \multirow[t]{2}{*}{1} & $\mathrm{n}$ & 69 & 8 & 0 & 0 & 77 \\
\hline & $\%$ & 89.6 & 10.4 & 0.0 & 0.0 & \\
\hline \multirow[t]{2}{*}{2} & $\mathrm{n}$ & 23 & 3 & 0 & 0 & 26 \\
\hline & $\%$ & 88.5 & 11.5 & 0.0 & 0.0 & \\
\hline \multirow[t]{2}{*}{3} & $\mathrm{n}$ & 154 & 45 & 9 & 9 & 209 \\
\hline & $\%$ & 73.7 & 21.5 & 4.3 & 4.3 & \\
\hline \multirow[t]{2}{*}{4} & $\mathrm{n}$ & 11 & 1 & 0 & 1 & 13 \\
\hline & $\%$ & 84.6 & 7.7 & 0.0 & 7.7 & \\
\hline \multirow[t]{2}{*}{5} & $\mathrm{n}$ & 31 & 9 & 10 & 8 & 58 \\
\hline & $\%$ & 53.4 & 15.5 & 17.2 & 13.8 & \\
\hline \multirow[t]{2}{*}{7} & $\mathrm{n}$ & 10 & 4 & 5 & 9 & 28 \\
\hline & $\%$ & 35.7 & 14.3 & 17.9 & 32.1 & \\
\hline \multirow[t]{2}{*}{8} & $\mathrm{n}$ & 10 & 6 & 6 & 2 & 24 \\
\hline & $\%$ & 41.7 & 25.0 & 25.0 & 8.3 & \\
\hline \multirow[t]{2}{*}{9} & $\mathrm{n}$ & 4 & 1 & 1 & 11 & 17 \\
\hline & $\%$ & 23.5 & 5.9 & 5.9 & 64.7 & \\
\hline \multirow[t]{2}{*}{10} & $\mathrm{n}$ & 3 & 0 & 2 & 5 & 10 \\
\hline & $\%$ & 30.0 & 0.0 & 20.0 & 50.0 & \\
\hline \multirow[t]{2}{*}{11} & $\mathrm{n}$ & 1 & 0 & 0 & 6 & 7 \\
\hline & $\%$ & 14.3 & 0.0 & 0.0 & 85.7 & \\
\hline \multirow[t]{2}{*}{$\Sigma$} & $\mathbf{n}$ & 316 & 77 & 31 & 45 & 469 \\
\hline & $\%$ & 67.4 & 16.4 & 6.6 & 9.6 & 100.0 \\
\hline
\end{tabular}

$n$ number, ductal carcinoma in situ, High-risk high-risk lesion 

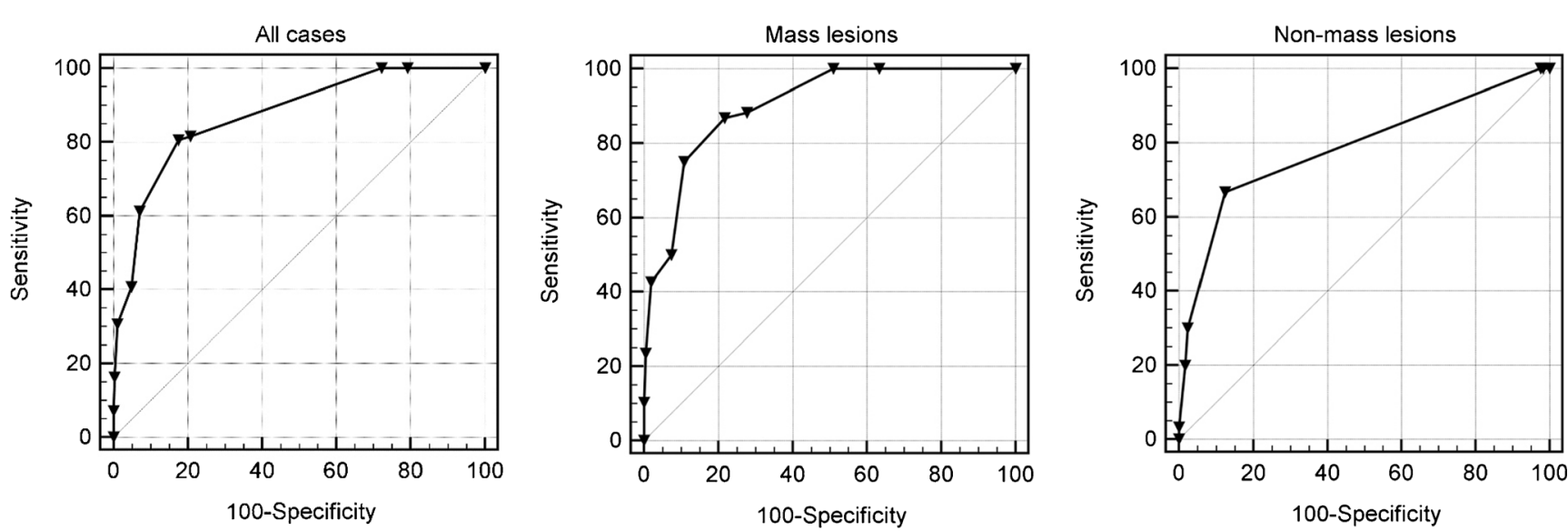

Fig. 6 Receiver operating characteristic (ROC) curves of all lesions included in the study, mass lesions and non-mass lesions. Details on the area under the curve (AUC) are given in the text and on diagnostic cut-off values in Tables 4, 5 and 6

was 0.873 (95\% CI: $0.839-0.901 ; P<0.0001$ ) (Fig. 6). Detailed results using the Tree flowchart in all lesions, masses or non-mass lesions, and details of different cutoff levels and their diagnostic parameters, are shown in Tables 4, 5 and 6. For mass lesions, the AUC for lesion diagnosis was 0.902 (95\% CI: $0.860-0.935 ; P<0.0001$ ), whereas in non-mass lesions the AUC was $0.786(95 \%$ CI: $0.722-0.841 ; P<0.0001$ ) (Fig. 6). MRI units by two vendors were used in this study: Siemens $(\mathrm{n}=383)$ and Philips $(\mathrm{n}=71)($ ESM 1). The diagnostic performance using the Tree algorithm did not differ between cases examined on either vendor's scanners (AUC Siemens = 0.880 , standard error $=0.019$; AUC Philips $=0.859$, standard error $=0.056 ; P=0.724)$.
Of 371 benign lesions, 103 (27.8\%) (99 mass and four nonmass lesions) could have been predicted using the Tree flowchart, with a cut-off of $\leq 2$ in mass and non-mass lesions ruling out malignancy, without gaining any false-negative results (NPV $=100 \%$ ). A cut-off of $\leq 2$ means that all lesions, but without the root sign and with persistent or plateau enhancement regardless of the lesion margins (smooth or irregular), would be considered benign.

A cut-off of $>8$ in the Tree flowchart to rule in malignancy would have predicted 30 of 98 true-positive findings (30.6\%), with a specificity of $98.9 \%$ and a positive likelihood ratio of 28.4 (Table 4). A cut-off of $>8$ includes all lesions showing washout with or without the root sign and lesions with plateau enhancement and the root sign.

Table 4 Diagnostic parameters and cut-off values of the Tree flowchart in all lesions included in the study

\begin{tabular}{|c|c|c|c|c|c|c|}
\hline \multicolumn{7}{|c|}{ All lesions } \\
\hline Cut-off & Sens & $95 \% \mathrm{CI}$ & Spec & $95 \% \mathrm{CI}$ & $+\mathrm{LR}$ & $-\mathrm{LR}$ \\
\hline$\geq 1$ & $100 \%(98 / 98)$ & $96.3-100$ & $0 \%(0 / 371)$ & $0-1$ & 1 & \\
\hline$>2$ & $100 \%(98 / 98)$ & $96.3-100$ & $27.8 \%(103 / 371)$ & $23.3-32.6$ & 1.38 & 0 \\
\hline$>3$ & $81.6 \%(80 / 98)$ & $72.5-88.7$ & $79.3 \%(294 / 371)$ & $74.8-83.3$ & 3.93 & 0.23 \\
\hline$>4$ & $80.6 \%(79 / 98)$ & $71.4-87.9$ & $82.5 \%(306 / 371)$ & $78.2-86.2$ & 4.6 & 0.24 \\
\hline$>5$ & $61.2 \%(60 / 98)$ & $50.8-70.9$ & $93 \%(345 / 371)$ & $89.9-95.4$ & 8.74 & 0.42 \\
\hline$>7$ & $40.8 \%(40 / 98)$ & $31.0-51.2$ & $95.2 \%(353 / 371)$ & $92.4-97.1$ & 8.41 & 0.62 \\
\hline$>8$ & $30.6 \%(30 / 98)$ & $21.7-40.7$ & $99 \%(367 / 371)$ & $97.3-99.7$ & 28.39 & 0.7 \\
\hline$>9$ & $16.3 \%(16 / 98)$ & $9.6-25.2$ & $99.7 \%(370 / 371)$ & $98.5-100$ & 60.57 & 0.84 \\
\hline$>10$ & $7.1 \%(7 / 98)$ & $2.9-14.2$ & $100 \%(371 / 371)$ & $99-100$ & & 0.93 \\
\hline$>11$ & $0 \%(0 / 98)$ & $0-3.7$ & $100 \%(371 / 371)$ & $99-100$ & & 1 \\
\hline
\end{tabular}

$n$ number, Ben benign, Mal malignant, Sens sensitivity, $C I$ confidence interval, Spec specificity, $+L R$ positive likelihood ratio, - LR negative likelihood ratio, $\sum$ sum 
Table 5 Diagnostic parameters and cut-off values of the Tree flowchart in mass lesions

Mass lesions

\begin{tabular}{|c|c|c|c|c|c|c|}
\hline Cut-off & Sens & $95 \% \mathrm{CI}$ & Spec & $95 \% \mathrm{CI}$ & $+\mathrm{LR}$ & $-\mathrm{LR}$ \\
\hline$\geq 1$ & $100 \%(68 / 68)$ & 94. 7-100 & $0 \%(0 / 202)$ & $0-1.8$ & 1 & \\
\hline$>2$ & $100 \%(68 / 68)$ & $94.7-100$ & $49 \%(99 / 202)$ & $41.9-56.1$ & 1.96 & 0 \\
\hline$>3$ & $88.2 \%(60 / 68)$ & $78.1-94.8$ & $72.3 \%(146 / 202)$ & $65.6-78.3$ & 3.18 & 0.16 \\
\hline$>4$ & $86.8 \%(59 / 68)$ & $76.4-93.8$ & $78.2 \%(158 / 202)$ & $71.9-83.7$ & 3.98 & 0.17 \\
\hline$>5$ & $75 \%(51 / 68)$ & $63-84.7$ & $89.1 \%(180 / 202)$ & $84-93$ & 6.89 & 0.28 \\
\hline$>7$ & $50 \%(34 / 68)$ & $37.6-62.4$ & $92.6 \%(187 / 202)$ & $88-95.8$ & 6.73 & 0.54 \\
\hline$>8$ & $42.7 \%(29 / 68)$ & $30.7-55.2$ & $98 \%(198 / 202)$ & $95-99.5$ & 21.54 & 0.59 \\
\hline$>9$ & $23.5 \%(16 / 68$ & $14.1-35.4$ & $99.5 \%(201 / 202)$ & $97.3-100$ & 47.53 & 0.77 \\
\hline$>10$ & $10.3 \%(7 / 68)$ & $4.2-20.1$ & $100 \%(202 / 202)$ & $98.2-100$ & & 0.9 \\
\hline$>11$ & $0 \%(0 / 68)$ & $0-5.3$ & $100 \%(202 / 202)$ & $98.2-100$ & & 1 \\
\hline
\end{tabular}

$n$ number, Ben benign, Mal malignant, Sens sensitivity, $C I$ confidence interval, Spec specificity, + LR positive likelihood ratio, - LR negative likelihood ratio, $\sum$ sum

\section{Inter-reader agreement}

Two readers independently read 82 consecutive cases. The kappa agreement among the two readers for the characterization of breast lesions according to the Tree flowchart was almost perfect ( $\mathrm{k}=0.944$ [95\% CI: 0.889-0.998]).

\section{Discussion}

Our study shows that using a simple classification system (the Tree flowchart), malignancy can be excluded in $27.8 \%$ of MRI-only lesions previously classified as BI-RADS 4, without resulting in any false-negative findings, thus leading to a substantial decrease in unnecessary biopsies. Therefore, the Tree flowchart holds the potential to reduce the number of costly and time-consuming MRI-guided biopsies, and therefore to decrease healthcare costs, patient discomfort and the risk of possible adverse effects due to the invasive procedure. The Tree flowchart is intuitive and suitable for readers of different levels of experience, and it is easily applicable in the routine clinical setting, as it requires standard breast MRI sequences only.

As the Tree flowchart is based on T2-weighted sequences and dynamic, contrast-enhanced, T1-weighted sequences that are generally recommended for every breast MRI, it does not require any additional imaging [2]. Of note, short tau inversion recovery (STIR) sequences are as suitable as T2-weighted TSE sequences for the assessment of oedema [28]. Furthermore, the Tree flowchart is based on simple dynamic and morphological features. Thus, its application does not require any extra reading

Table 6 Diagnostic parameters and cut-off values of the Tree flowchart in non-mass lesions.

\begin{tabular}{|c|c|c|c|c|c|c|}
\hline \multicolumn{7}{|c|}{ Non-mass lesions } \\
\hline Cut-off & Sens & $95 \% \mathrm{CI}$ & Spec & $95 \% \mathrm{CI}$ & $+\mathrm{LR}$ & -LR \\
\hline$\geq 1$ & $100 \%(30 / 30)$ & $88.4-100$ & $0 \%$ & $0-2.2$ & 1 & \\
\hline$>2$ & $100 \%(30 / 30)$ & $88.4-100$ & $2.37 \%(4 / 169)$ & $0.6-5.9$ & 1.02 & 0 \\
\hline$>3$ & $66.67 \%(20 / 30)$ & $47.2-82.7$ & $87.57 \%(148 / 169)$ & $81.6-92.1$ & 5.37 & 0.38 \\
\hline$>5$ & $30 \%(9 / 30)$ & $14.7-49.4$ & $97.63 \%(165 / 169)$ & $94.1-99.4$ & 12.67 & 0.72 \\
\hline$>7$ & $20 \%(6 / 30)$ & $7.7-38.6$ & $98.22 \%(166 / 169)$ & $94.9-99.6$ & 11.27 & 0.81 \\
\hline$>8$ & $3.33 \%(1 / 30)$ & $0.1-17.2$ & $100 \%(169 / 169)$ & $97.8-100$ & & 0.97 \\
\hline$>9$ & $0 \%(0 / 30)$ & $0-11.6$ & $100 \%(169 / 169)$ & $97.8-100$ & & 1 \\
\hline
\end{tabular}

$n$ number, Ben benign, Mal malignant, Sens sensitivity, $C I$ confidence interval, Spec specificity, $+L R$ positive likelihood ratio, - LR negative likelihood ratio, $\sum$ sum 
or computational time, as the additional acquisition of DWI would, for example [30, 31].

To date, lesion analysis based on the Tree flowchart does not include additional functional imaging, such as DWI or MR spectroscopy. Several studies have shown that DWI, as an adjunct to conventional breast MRI, holds the potential to reduce false-positive results and increase specificity with lower ADC values exhibited by malignant versus benign lesions [32-35]. In an analogous fashion, DWI may be combined with the Tree flowchart. Considering the current trend of combining information from multiple sources, referred to as Big Data, it is conceivable that more complex computer-assisted algorithms will be developed that rely not only on semantic and agnostic imaging features, but also on clinical background information as well as follow-up examinations. However, there are limitations concerning artifacts and spatial distortions, and different DWI approaches have prevented the determination of a generalizable ADC threshold value to distinguish malignant from benign lesions [34, 36-38].

The signs included in the Tree flowchart (root sign, enhancement kinetics, lesion margin, internal enhancement pattern and ipsilateral oedema) were selected initially based on their nonredundancy and the association with malignant breast lesions [23, 25-27]. More recent studies have proven the reliability and significance of these signs [17, 24, 39-41]. The Tree flowchart was initially evaluated in an exploratory study [23], and thereafter validated in a single clinical centre [24]. In this study, the Tree flowchart was applied to all patients undergoing MRIguided VABBs in a large tertiary breast-care centre, where patients present with imaging acquired either at the same centre or at other institutions for a second reading of their MRI. This setting allows the application of the Tree flowchart to images from different clinical centres. After the initial two studies on homogeneous patient cohorts, this study set out to test the practical application of this decision algorithm. In the daily practice of an assessment centre, breast radiologists need to deal with imaging of varying quality. This is a setting where the Tree flowchart can show its true potential to reduce unnecessary breast biopsies.

This study aimed to establish the diagnostic value of the Tree flowchart in suspicious lesions visible only on MRI in order to provide clinical guidance in this setting. Rather than the mere application of a single cut-off value at the left upper part of the ROC curve, which maximizes the sum of sensitivity and specificity, different cut-off values can be applied according to the population in question to rule-out or rule-in malignancy [42]. Marino et al. recommended a cut-off value of $\leq 4$ to rule-out malignancy in their cohort [24]. We identified a non-negligible number of malignant lesions in Tree scores 3 and 4 in our cohort of MRI-only lesions (false negative score 3 lesions comprised 9 DCIS, 8 IDC and one ILC and the only false negative score 4 lesion measured $4 \mathrm{~mm}$ on the MRI images and histopathology revealed a $1.5 \mathrm{~mm}$ invasive mucinous carcinoma). Although the number of invasive cancers in this group was very small at $4.5 \%$ (ten of 222), a cut-off of $\leq 2$ might be preferable in the investigated setting to achieve the highest possible sensitivity. In this respect, score 4 deserves further research as we only identified one malignant lesion in this small subgroup.

Both the MRI BI-RADS terminology and the Tree flowchart show higher diagnostic accuracy in mass lesions than in non-mass lesions [43-46]. In MRI-only mass lesions, the Tree flowchart improved diagnostic accuracy by correctly identifying 99 benign lesions out of 270 lesions previously classified as BI-RADS 4 (33.3\%) and four benign lesions out of 199 non-mass lesions $(2.0 \%)$.

This study shows that the Tree flowchart can guide decision-making after discrepant results between MRI-guided biopsy and imaging. A relevant rate of false-negative histopathological results are initially obtained by MRI-guided biopsy of suspicious breast lesions visible on MRI $(0-17 \%)[6,22$, 47-49]. In these cases, potential radiological-pathological mismatch needs to be assessed. With a test that would provide high specificity, such as the Tree flowchart, the radiologist may confidently request surgical biopsy after a negative histopathological result $[9,22]$. This is particularly the case for lesions assigned a Tree result of $>8$, resulting in a positive likelihood ratio of $>28$, and thus practically ruling in malignancy. A negative biopsy result in such a case should immediately be referred either to repeat-biopsy or open surgery.

Two radiologists with different experience in breast MRI evaluated lesions according to the Tree flowchart with almost perfect inter-reader agreement, demonstrating that the Tree flowchart is easily applicable.

The primary limitation of this study was that the standard MRI scans analysed in this study were acquired on different scanner types at different field strengths and with different sequence parameters. On the one hand, the different image qualities and imaging protocols may have limited imaging interpretation based on the Tree flowchart and may be one reason why the rate of malignancies in flowchart nodes 3 and 4 was found to be somewhat higher than that previously reported [24]. On the other hand, the inclusion of MRI scans of different image qualities shows the reliability of the Tree flowchart in a realistic and heterogeneous setting of MR images, as may be seen at any reference centre offering MRI-guided VABB to patients referred from other institutions. The high inter-reader agreement and the diagnostic accuracy reported in this setting for both mass and non-mass lesions prove the applicability of the Tree flowchart in this heterogeneous cohort. In addition, subgroup analysis did not show a significant influence of MRI vendor on the accuracy of the Tree flowchart, thus corroborating its robustness regarding imaging protocol variations. Furthermore, only histopathologically verified MRI-only lesions were included in this study. Thus, the results may not apply to a general population or to all lesions visible on MRI in a similar manner. Further studies will be necessary to evaluate the applicability of the Tree flowchart to different subpopulations. 
In conclusion, this study showed that the Tree flowchart, with a cut-off value of $\leq 2$, can reduce the number of biopsies in MRI-only lesions by as much as $27.8 \%$, with no falsenegative cases, thus potentially decreasing healthcare costs and patient discomfort.

Acknowledgements Open access funding provided by Medical University of Vienna.

\section{Compliance with ethical standards}

Guarantor The scientific guarantor of this publication is Pascal A.T. Baltzer

Conflict of interest The authors of this manuscript declare no relationships with any companies, whose products or services may be related to the subject matter of the article.

Statistics and biometry One of the authors has significant statistical expertise. No complex statistical methods were necessary for this paper.

Ethical approval Institutional Review Board approval was obtained.

Informed consent Written informed consent was waived by the Institutional Review Board.

Study subjects or cohorts overlap Some study subjects or cohorts have been previously reported in:

Spick C, Schernthaner M, Pinker K, et al. (2016) MR-guided vacuumassisted breast biopsy of MRI-only lesions: a single center experience. Eur Radiol. doi: 10.1007/s00330-016-4267-9, and Spick C, PinkerDomenig K, Rudas M, et al. (2014) MRI-only lesions: application of diffusion-weighted imaging obviates unnecessary MR-guided breast biopsies. Eur Radiol 24:1204-10. doi: 10.1007/s00330-014-3153-6

\section{Methodology • retrospective \\ - diagnostic study \\ - performed at one institution}

Open Access This article is distributed under the terms of the Creative Commons Attribution 4.0 International License (http:// creativecommons.org/licenses/by/4.0/), which permits unrestricted use, distribution, and reproduction in any medium, provided you give appropriate credit to the original author(s) and the source, provide a link to the Creative Commons license, and indicate if changes were made.

\section{References}

1. Mann RM, Kuhl CK, Kinkel K, Boetes C (2008) Breast MRI: guidelines from the European Society of Breast Imaging. Eur Radiol 18:1307-1318

2. Sardanelli F, Boetes C, Borisch B et al (2010) Magnetic resonance imaging of the breast: recommendations from the EUSOMA working group. Eur J Cancer 46:1296-1316

3. Reston VA (2013) The American College of Radiology (ACR) (2013) Breast Imaging Reporting and Data System Atlas (BIRADS $®$ Atlas)

4. Spick C, Baltzer PAT (2014) Diagnostic utility of second-look US for breast lesions identified at MR imaging: systematic review and meta-analysis. Radiology 273:401-409
5. Perlet C, Heywang-Kobrunner SH, Heinig A et al (2006) Magnetic resonance-guided, vacuum-assisted breast biopsy: results from a European multicenter study of 538 lesions. Cancer 106:982-990

6. Schrading S, Simon B, Braun M et al (2010) MRI-guided breast biopsy: influence of choice of vacuum biopsy system on the mode of biopsy of MRI-only suspicious breast lesions. AJR Am J Roentgenol 194:1650-1657

7. Crystal P, Sadaf A, Bukhanov K et al (2011) High-risk lesions diagnosed at MRI-guided vacuum-assisted breast biopsy: can underestimation be predicted? Eur Radiol 21:582-589

8. Fischer U, Schwethelm L, Baum FT et al (2009) Effort, accuracy and histology of MR-guided vacuum biopsy of suspicious breast lesions-retrospective evaluation after 389 interventions. RöFo Fortschritte auf dem Gebiete der Röntgenstrahlen und der Nukl 181:774-781

9. Floery D, Helbich TH (2006) MRI-Guided percutaneous biopsy of breast lesions: materials, techniques, success rates, and management in patients with suspected radiologic-pathologic mismatch. Magn Reson Imaging Clin N Am 14:411-25

10. Lee CI, Bensink ME, Berry K et al (2016) Performance goals for an adjunct diagnostic test to reduce unnecessary biopsies after screening mammography: analysis of costs, benefits, and consequences. J Am Coll Radiol 13:R81-R88

11. Spick C, Pinker-Domenig K, Rudas M et al (2014) MRIonly lesions: application of diffusion-weighted imaging obviates unnecessary MR-guided breast biopsies. Eur Radiol 24: $1204-1210$

12. Pinker K, Bogner W, Baltzer P et al (2014) Improved differentiation of benign and malignant breast tumors with multiparametric 18 fluorodeoxyglucose positron emission tomography magnetic resonance imaging: a feasibility study. Clin Cancer Res 20:3540-3549

13. Pinker K, Bogner W, Baltzer P et al (2014) Improved diagnostic accuracy with multiparametric magnetic resonance imaging of the breast using dynamic contrast-enhanced magnetic resonance imaging, diffusion-weighted imaging, and 3-dimensional proton magnetic resonance spectroscopic imaging. Invest Radiol 49:421-430

14. Montemezzi S, Cavedon C, Camera L et al (2016) 1H-MR spectroscopy of suspicious breast mass lesions at 3T: a clinical experience. Radiol Med. doi:10.1007/s11547-016-0713-7

15. Magometschnigg HF, Baltzer PA, Fueger B et al (2015) Diagnostic accuracy of 18F-FDG PET/CT compared with that of contrastenhanced MRI of the breast at 3 T. Eur J Nucl Med Mol Imaging 42:1656-1665

16. Pinker K, Helbich TH, Morris EA (2017) The potential of multiparametric MRI of the breast. Br J Radiol 90:20160715

17. Kim SG, Freed M, Leite APK et al (2016) Separation of benign and malignant breast lesions using dynamic contrast enhanced MRI in a biopsy cohort. J Magn Reson Imaging. doi:10.1002/jmri.25501

18. Ikeda DM, Hylton NM, Kinkel K et al (2001) Development, standardization, and testing of a lexicon for reporting contrast-enhanced breast magnetic resonance imaging studies. J Magn Reson Imaging 13:889-895

19. Kim SJ, Morris EA, Liberman L et al (2001) Observer variability and applicability of BI-RADS terminology for breast MR imaging: invasive carcinomas as focal masses. AJR Am J Roentgenol 177: $551-557$

20. Kinkel K, Helbich TH, Esserman LJ et al (2000) Dynamic highspatial-resolution MR imaging of suspicious breast lesions: diagnostic criteria and interobserver variability. AJR Am J Roentgenol 175:35-43

21. Riedl CC, Luft N, Bernhart C et al (2015) Triple-modality screening trial for familial breast cancer underlines the importance of magnetic resonance imaging and questions the role of mammography and ultrasound regardless of patient mutation status, age, and breast density. J Clin Oncol 33:1128-1135 
22. Spick C, Schernthaner M, Pinker K et al (2016) MR-guided vacuum-assisted breast biopsy of MRI-only lesions: a single center experience. Eur Radiol. doi:10.1007/s00330-016-4267-9

23. Baltzer PAT, Dietzel M, Kaiser WA (2013) A simple and robust classification tree for differentiation between benign and malignant lesions in MR-mammography. Eur Radiol 23:2051-2060

24. Marino MA, Clauser P, Woitek R et al (2015) A simple scoring system for breast MRI interpretation: does it compensate for reader experience? Eur Radiol. doi:10.1007/s00330-015-4075-7

25. Kaiser WA, Zeitler E (1989) MR imaging of the breast: fast imaging sequences with and without Gd-DTPA. preliminary observations. Radiology 170:681-686

26. Dietzel M, Baltzer PA, Vag T et al (2010) Differential diagnosis of breast lesions $5 \mathrm{~mm}$ or less. J Comput Assist Tomogr 34:456-464

27. Malich A, Fischer DR, Wurdinger S et al (2005) Potential MRI interpretation model: differentiation of benign from malignant breast masses. AJR Am J Roentgenol 185:964-970

28. Baltzer PAT, Gajda DM (2011) A systematic comparison of two pulse sequences for edema assessment in MR-mammography. Eur J Radiol. doi:10.1016/j.ejrad.2011.03.001

29. European Breast Cancer Network (EBCN) (2006) Quality assurance guidelines for pathology. In: Perry N, Broeders M, de Wolf C, et al (eds) Eur. Guidel. Qual. Assur. breast cancer Screen. diagnosis, 4th ed. pp 219-255

30. Bickel H, Pinker-Domenig K, Bogner W et al (2015) Quantitative apparent diffusion coefficient as a noninvasive imaging biomarker for the differentiation of invasive breast cancer and ductal carcinoma in situ. Invest Radiol 50:95-100

31. Pinker K, Bickel H, Helbich TH et al (2013) Combined contrastenhanced magnetic resonance and diffusion-weighted imaging reading adapted to the \&quot:Breast Imaging Reporting and Data System\&quot; for multiparametric 3-T imaging of breast lesions. Eur Radiol 23:1791-1802

32. Zhang L, Tang M, Min Z et al (2016) Accuracy of combined dynamic contrast-enhanced magnetic resonance imaging and diffusion-weighted imaging for breast cancer detection: a metaanalysis. Acta Radiol 57:651-660

33. Chen X, Li W, Zhang Y et al (2010) Meta-analysis of quantitative diffusion-weighted MR imaging in the differential diagnosis of breast lesions. BMC Cancer 10:693

34. Partridge SC, Nissan N, Rahbar H (2016) Diffusion-weighted breast MRI: Clinical applications and emerging techniques. J Magn Reson Imaging. doi:10.1002/jmri.25479

35. Baltzer A, Dietzel M, Kaiser CG, Baltzer PA (2016) Combined reading of contrast enhanced and diffusion weighted magnetic resonance imaging by using a simple sum score. Eur Radiol 26:884 891
36. Peters NHGM, Vincken KL, van den Bosch MAAJ et al (2010) Quantitative diffusion weighted imaging for differentiation of benign and malignant breast lesions: the influence of the choice of $b$ values. J Magn Reson Imaging 31:1100-1105

37. Le Bihan D, Poupon C, Amadon A, Lethimonnier F (2006) Artifacts and pitfalls in diffusion MRI. J Magn Reson Imaging 24:478-488

38. Partridge SC, DeMartini WB, Kurland BF et al (2009) Quantitative diffusion-weighted imaging as an adjunct to conventional breast MRI for improved positive predictive value. AJR Am J Roentgenol 193:1716-1722

39. Abe H, Mori N, Tsuchiya K et al (2016) Kinetic analysis of benign and malignant breast lesions with ultrafast dynamic contrastenhanced MRI: comparison with standard kinetic assessment. Am J Roentgenol 207:1159-1166

40. Kaiser CG, Herold M, Baltzer PAT et al (2015) Is 'Prepectoral Edema' a morphologic sign for malignant breast tumors? Acad Radiol 22:684-689

41. Wang L, Du J, Li F-H et al (2013) Diagnostic efficacy of contrastenhanced sonography by combined qualitative and quantitative analysis in breast lesions: a comparative study with magnetic resonance imaging. J Ultrasound Med 32:1805-1814

42. Altman DG, Bland JM (1994) Diagnostic tests 3: receiver operating characteristic plots. BMJ 309:188

43. Gutierrez RL, DeMartini WB, Eby PR et al (2009) BI-RADS lesion characteristics predict likelihood of malignancy in breast MRI for masses but not for nonmasslike enhancement. AJR Am J Roentgenol 193:994-1000

44. Benndorf M, Baltzer PAT, Kaiser WA (2011) Assessing the degree of collinearity among the lesion features of the MRI BI-RADS lexicon. Eur J Radiol 80:e322-e324

45. Jansen SA, Shimauchi A, Zak L et al (2011) The diverse pathology and kinetics of mass, nonmass, and focus enhancement on MR imaging of the breast. J Magn Reson Imaging 33:1382-1389

46. Baltzer PAT, Benndorf M, Dietzel M et al (2010) False-positive findings at contrast-enhanced breast MRI: a BI-RADS descriptor study. AJR Am J Roentgenol 194:1658-1663

47. Rauch GM, Dogan BE, Smith TB et al (2012) Outcome analysis of 9-gauge MRI-guided vacuum-assisted core needle breast biopsies. AJR Am J Roentgenol 198:292-299

48. Imschweiler T, Haueisen H, Kampmann G et al (2014) MRI-guided vacuum-assisted breast biopsy: comparison with stereotactically guided and ultrasound-guided techniques. Eur Radiol 24:128-135

49. Liberman L, Bracero N, Morris E et al (2005) MRI-guided 9-gauge vacuum-assisted breast biopsy: initial clinical experience. AJR Am J Roentgenol 185:183-193 